\title{
Prediction of mechanical properties of rail pads under in-service conditions through machine learning algorithms
}

\author{
Diego Ferreño ${ }^{(1)}$, Jose A. Sainz-Aja ${ }^{(1)}$, Isidro A. Carrascal ${ }^{(1)}$, Miguel Cuartas ${ }^{(2)}$, Joao Pombo ${ }^{(3,4)}$, \\ Jose A. Casado(1), Soraya Diego ${ }^{(1)}$
}

(1) LADICIM (Laboratory of Science and Engineering of Materials), University of Cantabria. E.T.S. de Ingenieros de Caminos, Canales y Puertos, Av. Los Castros 44, Santander, 39005, Spain.

(2) GTI (Group of Information Technologies), University of Cantabria. E.T.S. de Ingenieros de Caminos, Canales y Puertos, Av. Los Castros 44, 39005 Santander, Spain.
(3) Institute of Railway Research, University of Huddersfield, Queensgate, Huddersfield, HD1 3DG, UK.
(4) IDMEC, Instituto Superior Técnico, Universidade de Lisboa, Lisboa, Portugal and ISEL, IPL, Lisboa, Portugal.
*Corresponding author.

\section{Highlights}

- This study focuses on rail pads fabricated in EPDM, TPE and EVA

- Pads' stiffness was predicted through Machine Learning from the working conditions

- The importance of each in-service condition was ascertained

- The dependency of each working condition on the stiffness was estimated.

- An application capable of predicting rail pad stiffness was developed

\section{Abstract}

Train operations generate high impact and fatigue loads that degrade the rail infrastructure and the vehicle components. Rail pads are installed between the rails and the sleepers in order to damp the transmission of vibrations and noise and to provide flexibility to the track. These components play a crucial role in maximizing the durability of the railway assets and minimizing maintenance costs. Rail pads can be fabricated with different polymeric materials that exhibit non-linear mechanical behaviours, which strongly depend on the service conditions. Therefore, it is extremely difficult to estimate their mechanical properties, in particular the dynamic stiffness. In this work, several machine learning methodologies (multilinear regression, $\mathrm{K}$ nearest neighbours, regression tree, random forest, gradient boosting, multi-layer perceptron and support vector machine) were used to determine the dynamic stiffness of rail pads depending on their in-service conditions (temperature, frequency, axle load and toe load). 720 experimental tests, under different realistic operating conditions, were performed to produce a dataset that was then used for the training and testing of the machine learning methods. The optimal algorithm was gradient boosting for EPDM $\left(R^{2}\right.$ of 0.995 and mean absolute percentage error of $5.08 \%$ in the test dataset), TPE (0.994 and $2.32 \%)$ and EVA (0.968 and $4.91 \%)$ pads. This model was implemented in an application, available for the readers of this journal, developed on the Microsoft .Net platform that allows the dynamic stiffness of the pads study to be estimated as a function of the temperature, frequency, axle load and toe load.

Keywords: Railway dynamics; Sleeper pads; Machine learning; Rail service conditions; Dynamic stiffness. 


\section{Introduction}

Given present trends, global demand for transport will more than double by 2050. The transport sector is currently responsible for around one quarter of the global $\mathrm{CO}_{2}$ emissions and such growth will result in greater emissions unless profound changes are made in transportation systems to achieve energy transitions globally. Rail is considered as one of the most energy efficient modes of transport for freight and passengers and, as a consequence, it provides a means to reduce the energy use and the environmental impact associated with transport. According to the International Energy Agency [1], the rail sector carries $8 \%$ of the world's passengers and $7 \%$ of global freight, but it represents only $2 \%$ of total transport energy demand.

The reduction of operation and maintenance (O\&M) costs and the minimization of incidents, which cause service delays and traffic disruption, brings very important challenges for rail transport in order to address modern society demands and to increase its attractiveness and competitiveness with respect to other means of transportation. A number of studies have been developed to understand the influence of train operations on the response of the infrastructure [2-10]. Other authors have devoted their attention to the detailed representation of the vehicletrack interaction using co-simulation methodologies encompassing multibody and finite element formulations [11]. These developments enable the integration of more detailed wheelrail contact models [12-21], to consider track irregularities $[22,23]$ and other track singularities [24-27] in the studies aiming to assess track performance and degradation evolution [28-31] under realistic operation conditions. All these studies contribute to the development of novel technology and to the reduction of O\&M costs.

Rail pads are the elements with the greatest influence on the track stiffness, which is a fundamental parameter for the maintenance of the track [32-38], in particular in the case of ballastless track $[39,40]$. One of the main difficulties encountered when developing a reliable numerical model of the mechanical response of the rail track is that the stiffness of the pads is highly influenced by the operating conditions. Although the specific influence is strongly material-dependent, some common trends have been experimentally described in the literature: stiffness increases with frequency [41-43], axle load [44] or toe load [42], and decreases with temperature [43]. Frequency can be as high as $80 \mathrm{kHz}$ [45], but frequencies above $20 \mathrm{~Hz}$ are considered as the result of track imperfections associated with small load amplitude values. Even though rail pads have a fundamental role in the attenuation of this type of frequencies, this work focuses on frequencies below $20 \mathrm{~Hz}$, since this range is representative of railway transportation and are associated with the highest load amplitudes. Testing at extreme temperatures can seriously modify the response of the component, but since the railway superstructure works at environmental temperatures, the characterization has been conducted in the range between -35 and $52 \stackrel{\circ}{\circ}$.

Obtaining a reliable numerical prediction of the dynamic stiffness is an unresolved issue, not only because of the highly non-linear behaviour of these polymeric materials but also because of the interactions existing between the aforementioned variables [41,42,44,46-48]. Machine Learning (ML) methods are particularly well-suited for nonlinear and highly interactive data modelling. ML algorithms build a mathematical model based on a training dataset to make predictions on fresh data (testing dataset) without being explicitly programmed to perform the task.

In this study, a number of ML algorithms are developed to predict the dynamic stiffness of three types of commercial rail pads, EPDM, TPE and EVA, as a function of the in-service conditions, 
namely frequency, axle load, temperature and toe load. The dataset comprised 720 experimental tests. The following seven algorithms were implemented for each material [49]: Multi-Linear Regression (MLR), K Nearest Neighbours (KNN), Regression Tree (RT), Random Forest (RF), Gradient Boosting (GB), Multi-Layer Perceptron (MLP) [50] and Support Vector Machine (SVM) [51,52]. 80\% of the observations are randomly chosen to form the training dataset. The algorithms are optimised through three-fold cross-validation. The test dataset $20 \%$ of the cases) was used to obtain an unbiased estimation of the predictive ability of the algorithms. Specific ML methods are implemented to sort, for each of the three types of pads, the operating conditions based on their relevance and to determine the specific influence of each variable on the dynamic stiffness.

The reliable ML algorithms proposed here can be used for a large number of applications. First, they can be a valuable tool for the definition of the type of rail pad to use, depending on the operating conditions. In addition, they can be very useful for the evaluation of the track conditions, increasing durability and providing information for maintenance management procedures. In this sense, these tools can be easily linked to finite element models of the infrastructure [53] in order to obtain the stress state on the track components depending on; the loads generated by the train service, the environmental conditions and the assembly settings. In this study, once the ML model with the best predictive ability had been obtained, an application to estimate the dynamic stiffness of the EPDM, TPE and EV pads characterized as a function of the operation conditions (frequency, axle load, temperature and toe load) was developed. This app is freely available for readers.

The remainder of the paper is organized as follows. The materials and experimental techniques are described in section 2. The ML methods, the regression algorithms and the techniques implemented to identify the most relevant features as well as their influence on the dynamic stiffness are detailed in section 3 . Section 4 is devoted to presenting the results of the analysis, their interpretation and the characteristics of the app. Finally, the conclusions of the research are summarized in section 5 .

\section{Materials and Experimental Tests}

\subsection{Materials}

In this study, three of the most commonly used materials for rail pads were selected, namely; EPDM, TPD and EVA, see Figure 1.

a) EPDM: ethylene-propylene copolymer, solid rail pad of $7 \mathrm{~mm}$ thickness without protrusions, with a hardness of 21 HS-D. This rail pad is used, for example, in Saudi Arabia's high-speed railways.

b) TPE: Polyester elastomer thermopolymer (Hytrel ${ }^{\mathrm{TM}}$ ), with oblong shaped protrusions, a thickness of $7 \mathrm{~mm}$, medium stiffness and a hardness of 47 HS-D. This is adopted, e.g., in the Spanish high-speed railways.

c) EVA: Ethylene-vinyl acetate, solid rail pad of $6 \mathrm{~mm}$ thickness without protrusions and a hardness of 46 HSD. This rail pad forms part of the solution adopted, for example, in the first Spanish high-speed line between Madrid and Sevilla. 


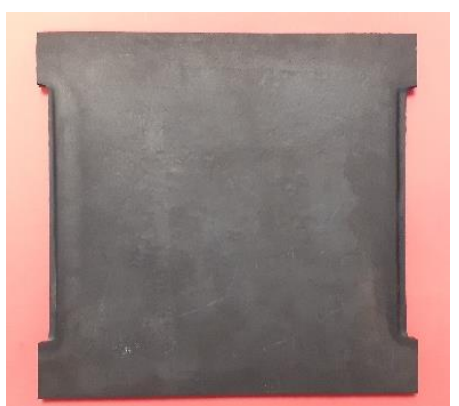

(a)

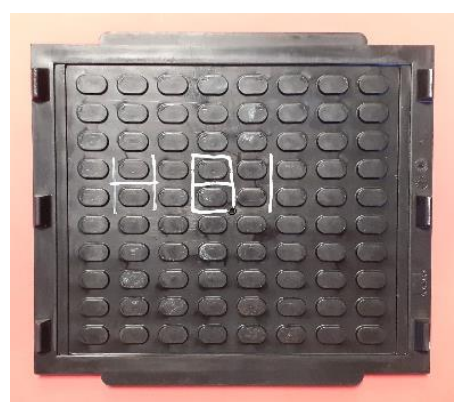

(b)

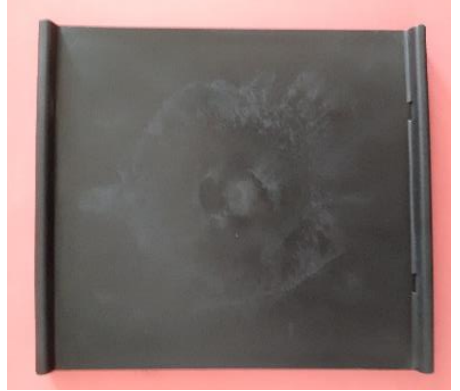

(c)
125

Figure 1.

Rail pads studied: (a) EPDM, $7 \mathrm{~mm}$ thick; (b) TPD, $7 \mathrm{~mm}$ thick; (c) EVA, $6 \mathrm{~mm}$ thick

\subsection{Experimental Campaign}

The procedures defined in standards EN 13481-2 [54] and EN 13146-9 [55] are used here to obtain experimentally the dynamic stiffness $\left(k_{d y n}\right)$ of the rail pads under different service conditions. The procedure is schematically depicted in Figure 2(a) where 1000 sinus-type cycles are applied between $F_{\min }$ and $F_{\max }$. Then, 10 cycles belonging to the last 100 cycles are selected and the average values of forces and displacements $\left(\overline{F_{\max }}, \overline{F_{m i n}}, \overline{D_{\max }}, \overline{D_{\min }}\right)$ are obtained as shown in Figure 2(b). Finally, the following equation is applied to obtain the dynamic stiffness:

$$
k_{\text {dyn }}=\frac{\overline{F_{\text {max }}}-\overline{F_{\text {min }}}}{\overline{D_{\max }}-\overline{D_{\min }}}
$$

To simulate different assembly and operating conditions of the pads, the values of frequency, load amplitude, temperature and toe load are varied, as shown in Table 1. $F_{\text {min }}$ in Figure 2(a) corresponds to the toe load and $F_{\max }$ is obtained by using the values of force amplitude defined in EN 13481-2 [54], namely 15.5, 21 and $31.5 \mathrm{kN}$. This standard also proposes three values for the frequency; 5,10 and $20 \mathrm{~Hz}$. In this study, a frequency of $2.5 \mathrm{~Hz}$ is also considered, as suggested by previous studies $[5,56]$. The Spanish standard for the definition of action on bridges considers as the minimum and maximum temperatures $-35 \circ \mathrm{C}$ and $52 \circ \mathrm{C}$, respectively, and the EN 13481-2 [54] defines the dynamic stiffness test temperature as $23 \pm 5$ o $\mathrm{C}$; to obtain regular intervals between temperatures, $-35 \stackrel{\circ}{\circ} \mathrm{C},-20 \stackrel{\circ}{\circ}, 0 \circ \mathrm{C}, 20^{\circ} \mathrm{C}$ and $52 \stackrel{\circ}{\circ} \mathrm{C}$ are considered in this research. To obtain regular intervals between temperatures, 0 으 $\mathrm{C}$ and $-20 \circ \mathrm{C}$ are also considered. Four values for the toe load are also analyzed here. These correspond to the reference value (18 $\mathrm{kN})$, representing a correct assembly of the fastening system, scenarios corresponding to the fracture of the system $(1 \mathrm{kN})$, an under-tightening $(9 \mathrm{kN})$ or an over-tightening $(25 \mathrm{kN})$. Since the experimental campaign on the three pads is carried out for four frequencies, three load amplitudes, five temperatures and four toe loads, it produces $3 \times 4 \times 3 \times 5 \times 4=720$ test results. To the best of the authors' knowledge, this is the most extensive and ambitious experimental research conducted in this field. 


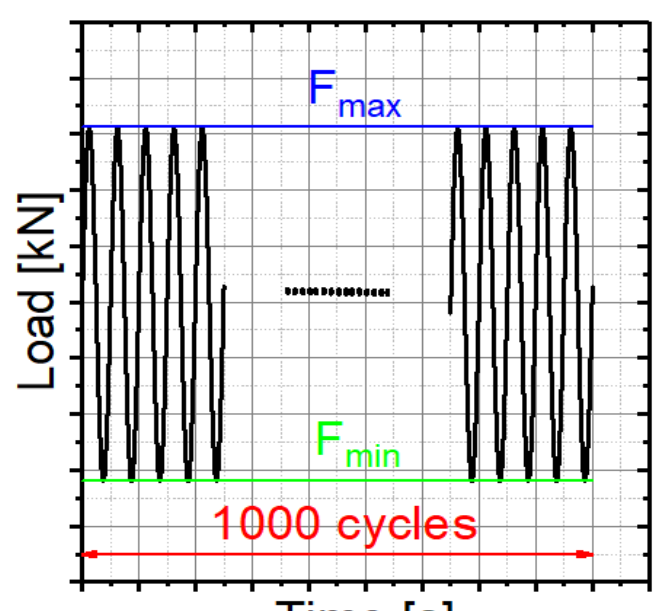

Time [s]

(a)

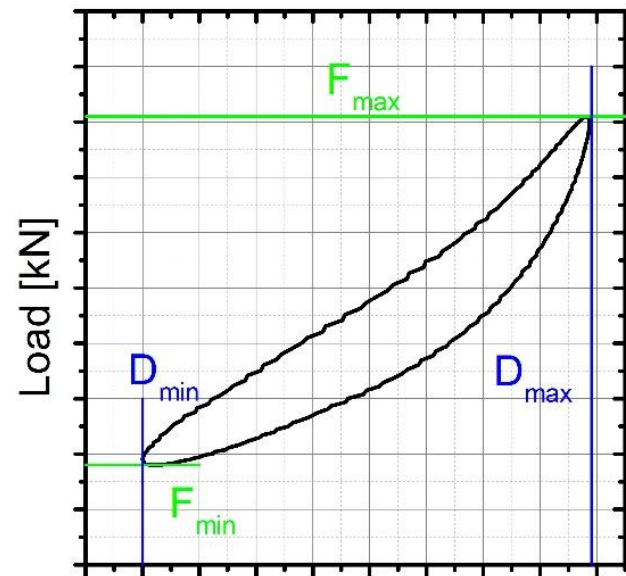

Displacement [mm]

(b)
Figure 2.

(a) Experimental tests to determine the dynamic stiffness. (b)
to obtain the dynamic stiffness

Table 1. Experimental parameters with influence on the mechanical response of the rail pads

\begin{tabular}{cccc}
\hline Frequency $[\mathrm{Hz}]$ & Amplitude $[\mathrm{kN}]$ & Temperature $\left[{ }^{\circ} \mathrm{C}\right]$ & Toe load $[\mathrm{kN}]$ \\
\hline 2.5 & 15.5 & -35 & 1 \\
5 & 21.0 & -20 & 9 \\
10 & 31.5 & 0 & 18 \\
20 & & 20 & 25 \\
& & 52 & \\
\hline
\end{tabular}

\section{Machine Learning Methods}

\subsection{Machine Learning Algorithms}

The ML algorithms are developed and evaluated in Python [57] using the libraries Numpy [58], Pandas [59], Scikit-learn [60], Matplotlib [61] and Seaborn [62]. The dataset consists of 720 instances and five features: material, frequency, load amplitude, toe load and temperature. The first variable, material, is categorical, i.e., it contains labels ('EPDM', 'TPE', 'EVA') rather than numeric values. Many ML methods cannot operate on categorical data but require all variables to be numeric. For this reason, the feature 'material' is one-hot encoded before any calculation.

Before processing, the five features are standardized to make them have zero-mean and unitvariance (StandardScaler). This technique is generally recommended and mandatory when employing some specific algorithms [63]. The following regression algorithms have been implemented in this research: Multiple Linear Regression (MLR), K-Nearest Neighbors (KNN), Regression Tree (RT), Random Forest (RF), Gradient Boosting (GB), Support Vector Machine (SVM), and Artificial Neural Networks (ANNs, in this case, Multi-Layer Perceptron, MLP). In the following, a brief description of these algorithms is presented:

- MLR is considered as a baseline algorithm for regression, i.e., a simple model which has a reasonable chance of providing decent results. In MLR, the relationship between the predictors (features) and the target / response variable is modeled through a linear equation which is fitted to the observed data. The linear nature of MLR restricts its ability to describe complex data. 
- In KNN, classification or regression are conducted for a new observation by summarizing the output variable of the ' $\mathrm{K}$ ' closest observations (the neighbors) with uniform weights or with weights proportional to the inverse of the distance from the query point. Classification is carried out using the mode of the neighbors while regression is usually based on the mean. The distance between instances is expressed through the Minkowski metric which depends on the power parameter, ' $p$ '. When $p=1$, this is equivalent to using the Manhattan distance and for $p=2$ the Euclidean distance.

- A decision tree is a flowchart-like structure in which each node splits a feature in two branches until a leaf node is reached which represents a class label or a numeric value, depending on whether a classification or regression is being conducted. In RTs, the cost function that is minimized to choose split points is the sum squared error of the samples in the training dataset. The most important advantage of RTs is that they are easy to understand and to interpret. The drawback is that they easily tend toward overfitting. To avoid overfitting, several pruning techniques are available.

- SVM was originally designed as a classifier [64] but may also be used for regression and feature selection [65]. In classification, SVM determines the optimal separating hyperplane between linearly separable classes maximizing the margin, which is defined as the distance between the hyperplane and the closest points on both sides (the support vectors). For nonperfectly separable classes, SVM must be modified to allow some points to be misclassified, which is achieved by introducing a "soft margin" [66]. Datasets that are highly nonlinear may in some cases be (linearly) separated after being (nonlinearly) mapped into a higher dimensional space [67]. This mapping gives rise to the kernel, which can be chosen by the user among different options such as linear, sigmoid, Gaussian or polynomial. The appropriate kernel function is selected by trial and error on the test set. In this case, SVM is referred to as kernelized SVM.

- Ensemble methods combine multiple "weak classifiers" into a single "strong classifier". A weak classifier is a classifier that performs poorly, but performs better than random guessing. Ensemble methods are classified into bagging-based and boosting-based, which are designed to reduce variance and bias, respectively. Bagging stands for Bootstrap Aggregating. RF is a widely used bagging method based on classification trees (weak learner). In RFs, each tree in the ensemble is built from a bootstrap sample of the training set. In addition, instead of using all the features, a random subset of features is selected, further randomizing the tree. In boosting, each new tree is a fit on a modified version of the original data set. Gradient Boosting trains many models in a sequential manner identifying the shortcomings of weak learners using gradients in the loss function.

- ANNs are mostly used for data classification and pattern recognition [68]. A basic ANN contains a large number of neurons / nodes arranged in layers. MLP contains one or more hidden layers (apart from one input and one output layer). The nodes of consecutive layers are connected and these connections have weights associated with them. In a feedforward network, the information moves in one direction from the input nodes, through the hidden nodes (if any) to the output nodes. The output of every neuron is obtained by applying an activation function to the linear combination of inputs (weights) to the neuron. Sigmoid, tanh and ReLu (Rectified Linear Unit) are the most widely used activation functions. MLPs are trained through the backpropagation algorithm. Gradient descent, Newton, conjugate gradient and Levenberg-Marquardt are different algorithms used to train an ANN.

$20 \%$ of the observations are randomly extracted to form a test dataset that was used to provide an unbiased evaluation of the models. Models are trained and the hyper-parameters are refined 
using the remaining $80 \%$ of the data. 3-fold cross-validation (CV3) was used for training and validation to guarantee the ability of the algorithm to generalize and to avoid overfitting. Parameter selection and model evaluation was conducted with GridSearchCV [69].

\subsection{Feature Importance and Permutation Importance}

The assessment of feature relevance provides insight into which factors most influence the output [70]. Feature Importance (FI) and Permutation Importance (PI) are two independent methods which only require an estimator that has been satisfactorily fitted. Both techniques are implemented in Scikit-Learn. Tree-based models, such as RF and GB, provide the Fl score, which is based on the mean decrease in impurity after a split. The PI of a feature is measured as the decrease in the model score when a single feature value is randomly shuffled. Thus, the larger the drop is in the score, the higher is the relevance of the feature. The amount of randomness in $\mathrm{Fl}$ or $\mathrm{PI}$ is calculated by repeating the process at random several times.

\subsection{Partial Dependence Plots}

A one-way Partial Dependence Plot (PDP) is a graph that displays the relationship between one feature and the target response. The $x$-axis represents the values of the feature, while the $y$-axis displays the partial dependence. PDPs are calculated using a model that has been previously fitted. For each value of the target feature, the PDP is obtained after marginalizing the predictions of the fitted model over the actual values of the rest of the features. Then, a distribution of values is obtained (one value for each of the instances of the dataset). This operation is repeated at intervals of values in the range of the target feature. Scikit-Learn provides a specific function to create a PDP.

\section{Results and Discussion}

\subsection{Selection of the Optimal Model for each Material}

The coefficient of determination, $R^{2}$, is selected as the regression score function for the optimization of the hyperparameters of the algorithms. These hyperparameters are listed in Table 2.

Table 2. Hyperparameters obtained after optimizing each ML algorithm for each pad

\begin{tabular}{|l|l|}
\hline MLR & N/A. \\
\hline KNN & EPDM: 'metric': 'manhattan', 'n_neighbors': 2, 'weights': 'uniform'. \\
& TPE: 'metric': 'manhattan', 'n_neighbors': 2, 'weights': 'uniform'. \\
& EVA: 'metric': 'manhattan', 'n_neighbors': 4, 'weights': 'distance'. \\
\hline RT & EPDM: 'criterion': 'mse', 'max_depth': 10, 'min_samples_leaf': 2, \\
& 'min_samples_split': 5, 'splitter': 'best'. \\
& TPE: 'metric': 'manhattan', 'n_neighbors': 2, 'weights': 'uniform'. \\
& EVA: 'criterion': 'mse', 'max_depth': 10, 'min_samples_leaf': 2, \\
& 'min_samples_split': 5, 'splitter': 'best'. \\
\hline RF & EPDM: 'bootstrap': False, 'max_depth': 15, 'max_features': 3, \\
& 'min_samples_split': 2, 'n_estimators': 5. \\
& TPE: 'bootstrap': True, 'max_depth': 10, 'max_features': 3, \\
& 'min_samples_split': 2, 'n_estimators': 50. \\
& EVA: 'bootstrap': True, 'max_depth': 15, 'max_features': 3, \\
& 'min_samples_split': 2, 'n_estimators': 10. \\
\hline GB & EPDM: 'learning_rate': 0.5, 'max_depth': 6, 'max_features': 3, \\
& 'min_samples_leaf': 5, 'min_samples_split': 2, 'n_estimators': 100. \\
\hline
\end{tabular}




\begin{tabular}{|c|l|}
\hline & TPE: 'learning_rate': 0.1, 'max_depth': 10, 'max_features': 3, \\
'min_samples_leaf': 5, 'min_samples_split': 10, 'n_estimators': 500. \\
EVA: 'learning_rate': 0.5, 'max_depth': 2, 'max_features': 3, \\
'min_samples_leaf': 10, 'min_samples_split': 3, 'n_estimators': 500. \\
\hline MLP & EPDM: 'activation': 'relu', 'alpha': 0.005, 'hidden_layer_sizes': (10, 10, 10), \\
& 'max_iter': 50000, 'solver': 'Ibfgs'. \\
& TPE: activation='relu', alpha=0.005, hidden_layer_sizes=(10, 10), \\
& max_iter=100000, solver='Ibfgs'. \\
& EVA: 'activation': 'relu', 'alpha': 0.0001, 'hidden_layer_sizes': $(10,10)$, \\
& 'max_iter': 25000, 'solver': 'adam'. \\
\hline SVM & EPDM: 'degree': 2, 'gamma': 'auto', 'kernel': 'linear'. \\
& TPE: 'degree': 2, 'gamma': 'auto', 'kernel': 'linear'. \\
& EVA: 'degree': 2, 'gamma': 'auto', 'kernel': 'linear'.
\end{tabular}

251 After optimization, the coefficient of determination $\left(\mathrm{R}^{2}\right)$, Root Mean Square Error (RMSE), Mean 252 Absolute Error (MAE) and Mean Absolute Percentage Error (MAPE) are calculated in the test dataset. This information is shown in Table 3, Table 4 and Table 5 for EPDM, TPE and EVA pads, respectively. As can be seen, GB stands out above the rest of the algorithms for all three materials. For EPDM and TPE, $\mathrm{R}^{2}$ is 0.995 while for EVA it is 0.988 . Also note that, for the selected algorithms, the MAPE is between $2.3 \%$ and $6.5 \%$.

Table 3. Statistical scores in the test dataset provided by each model for the EPDM pad

\begin{tabular}{ccccc}
\hline Algorithm & $\mathrm{R}^{2}$ & RMSE $[\mathrm{kN} / \mathrm{mm}]$ & $\mathrm{MAE}[\mathrm{kN} / \mathrm{mm}]$ & $\mathrm{MAPE}[\%]$ \\
\hline MLR & 0.452 & 100.39 & 133.67 & 88.78 \\
KNN & 0.628 & 61.58 & 110.14 & 34.76 \\
RT & 0.923 & 22.75 & 50.07 & 9.24 \\
RF & 0.965 & 16.45 & 33.66 & 6.18 \\
GB & 0.995 & 12.36 & 7.39 & 5.08 \\
MLP & 0.990 & 10.23 & 17.96 & 6.51 \\
SVM & 0.060 & 81.28 & 175.13 & 30.44 \\
\hline
\end{tabular}

258

Table 4. Statistical scores in the test dataset provided by each model for the TPE pad

\begin{tabular}{ccccc}
\hline Algorithm & $\mathrm{R}^{2}$ & $\mathrm{RMSE}[\mathrm{kN} / \mathrm{mm}]$ & $\mathrm{MAE}[\mathrm{kN} / \mathrm{mm}]$ & $\mathrm{MAPE}[\%]$ \\
\hline MLR & 0.759 & 40.18 & 52.02 & 17.83 \\
KNN & 0.872 & 27.73 & 37.99 & 10.18 \\
RT & 0.969 & 12.48 & 18.52 & 4.72 \\
RF & 0.977 & 9.86 & 16.18 & 3.45 \\
GB & 0.995 & 7.79 & 5.41 & 2.25 \\
MLP & 0.994 & 5.8 & 8.02 & 2.32 \\
SVM & 0.545 & 42.2 & 71.49 & 13.99 \\
\hline
\end{tabular}

Table 5. Statistical scores in the test dataset provided by each model for the EVA pad

\begin{tabular}{ccccc}
\hline Algorithm & $\mathrm{R}^{2}$ & $\mathrm{RMSE}[\mathrm{kN} / \mathrm{mm}]$ & $\mathrm{MAE}[\mathrm{kN} / \mathrm{mm}]$ & $\mathrm{MAPE}[\%]$ \\
\hline MLR & 0.928 & 76.62 & 93.24 & 7.88 \\
KNN & 0.927 & 67.93 & 93.64 & 7.04 \\
RT & 0.922 & 75.15 & 97.02 & 7.17 \\
RF & 0.968 & 50.23 & 62.52 & 4.91 \\
\hline
\end{tabular}




\begin{tabular}{ccccc}
\hline GB & 0.988 & 37.36 & 20.37 & 2.38 \\
MLP & 0.927 & 78.3 & 93.72 & 7.97 \\
SVM & 0.56 & 188.92 & 230.44 & 22.85 \\
\hline
\end{tabular}

262

263 Figure 3, Figure 4 and Figure 5 illustrate the prediction ability of the different ML algorithms for EPDM, TPE and EVA pads, respectively. The experimental values of stiffness are represented on the $\mathrm{X}$-axis and the predictions of each of the regressors are shown on the $\mathrm{Y}$-axis. Each graph includes a 1:1 line (corresponding to a perfect fitting) as well as two confidence bands separated from the centre line by a distance equal to the RMSE defined in Table 3, Table 4 and Table 5.
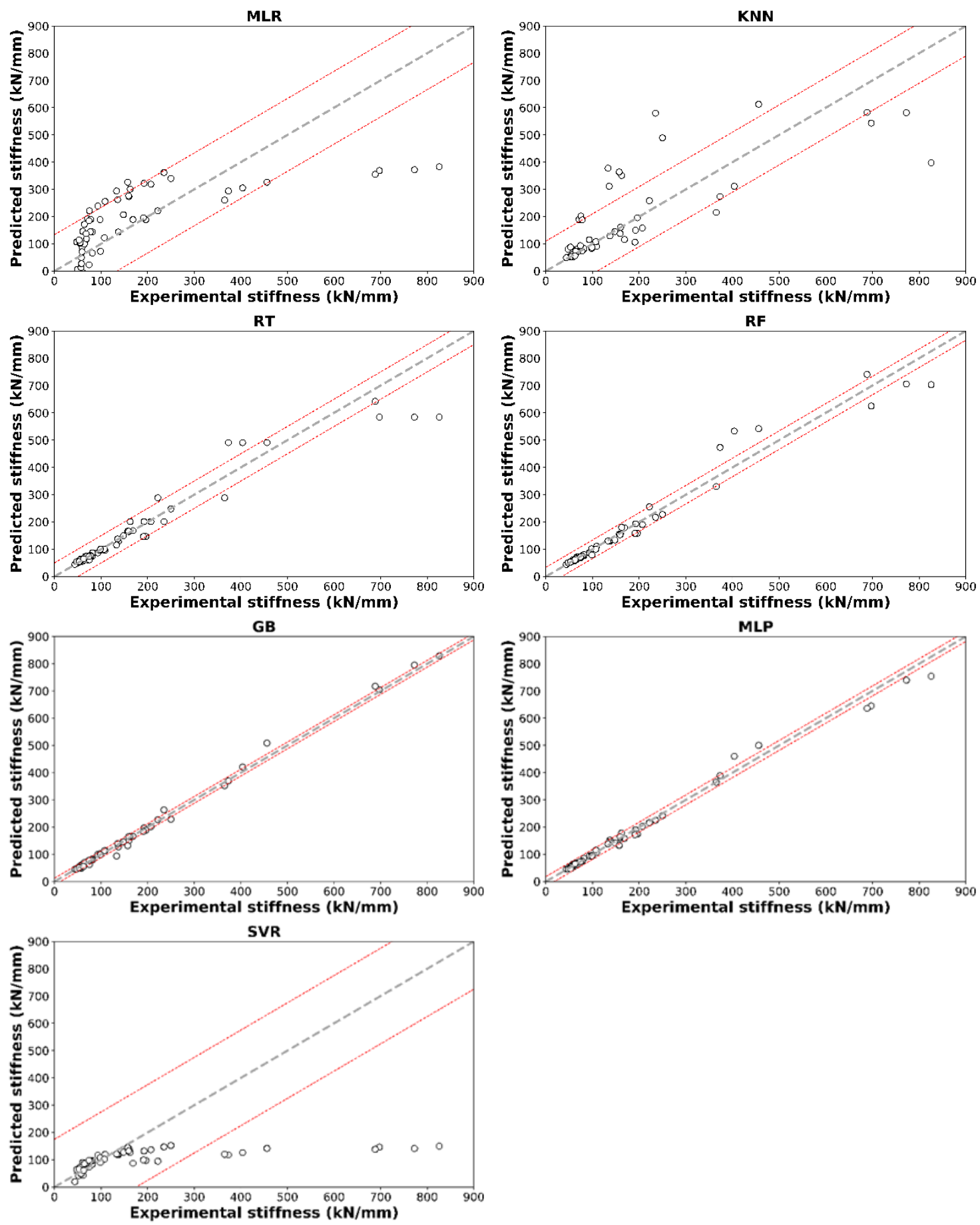

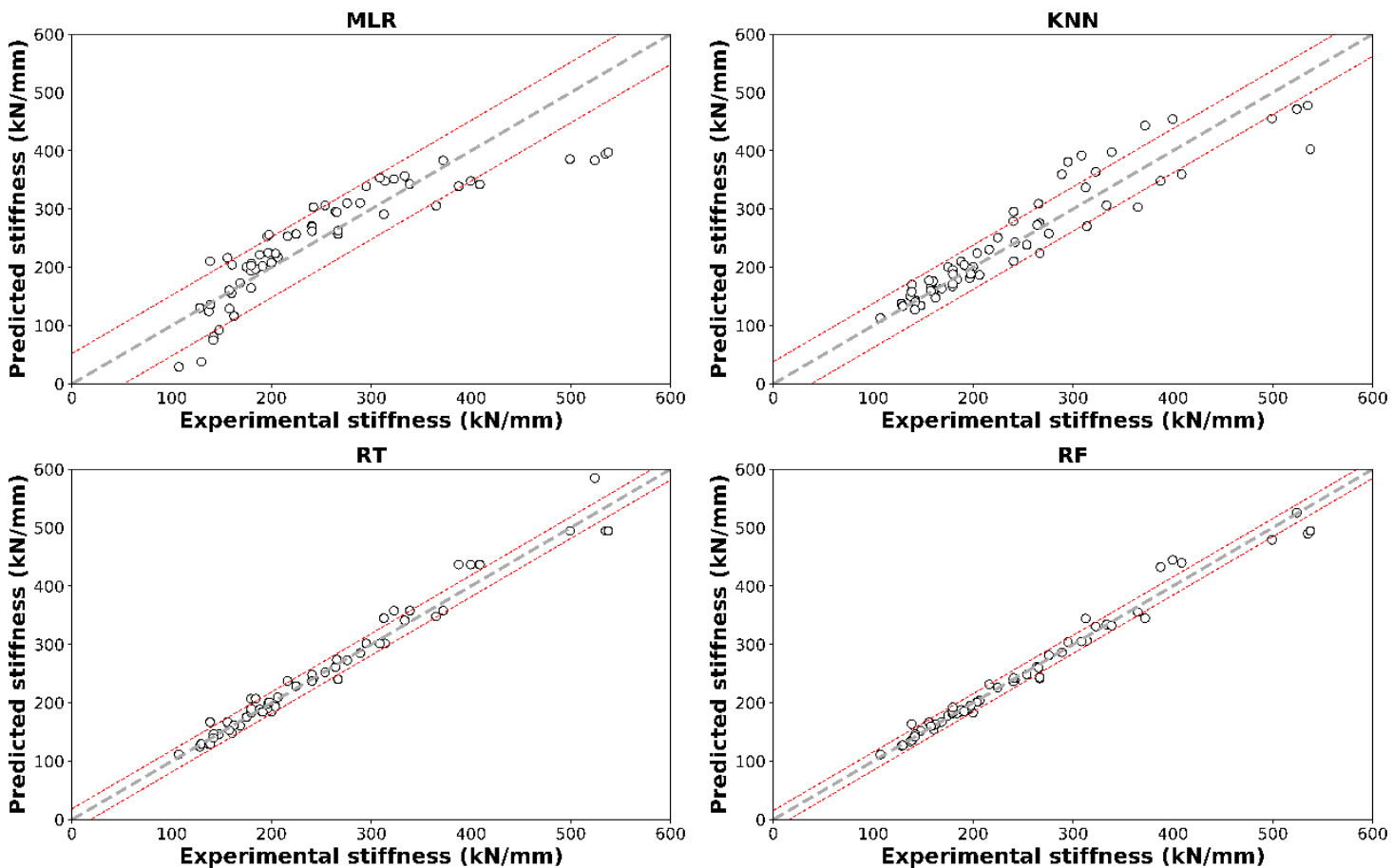

GB
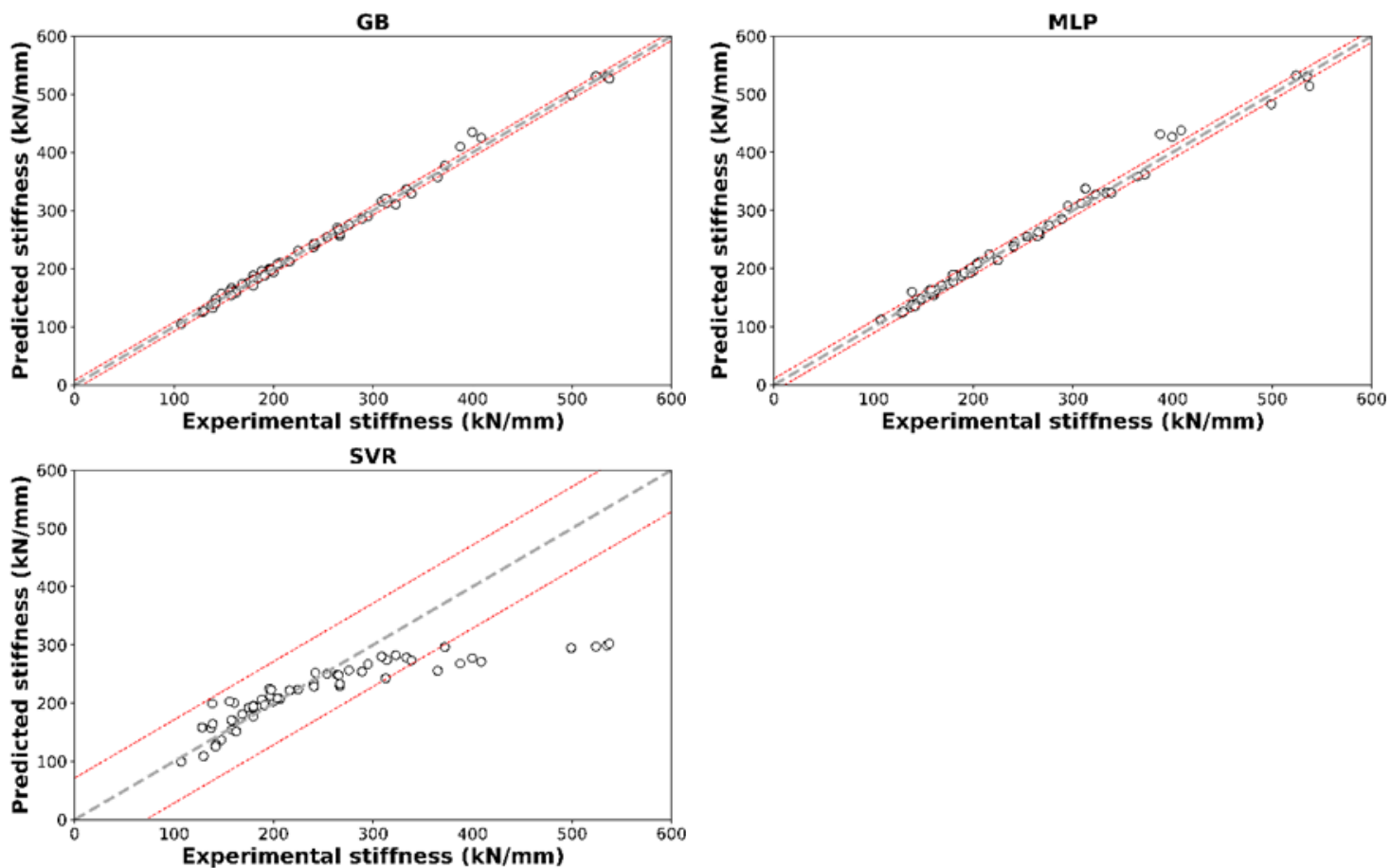

272

Figure 4. Comparison between the experimental and the predicted stiffness for each of the ML models optimized for the TPE rail pad 

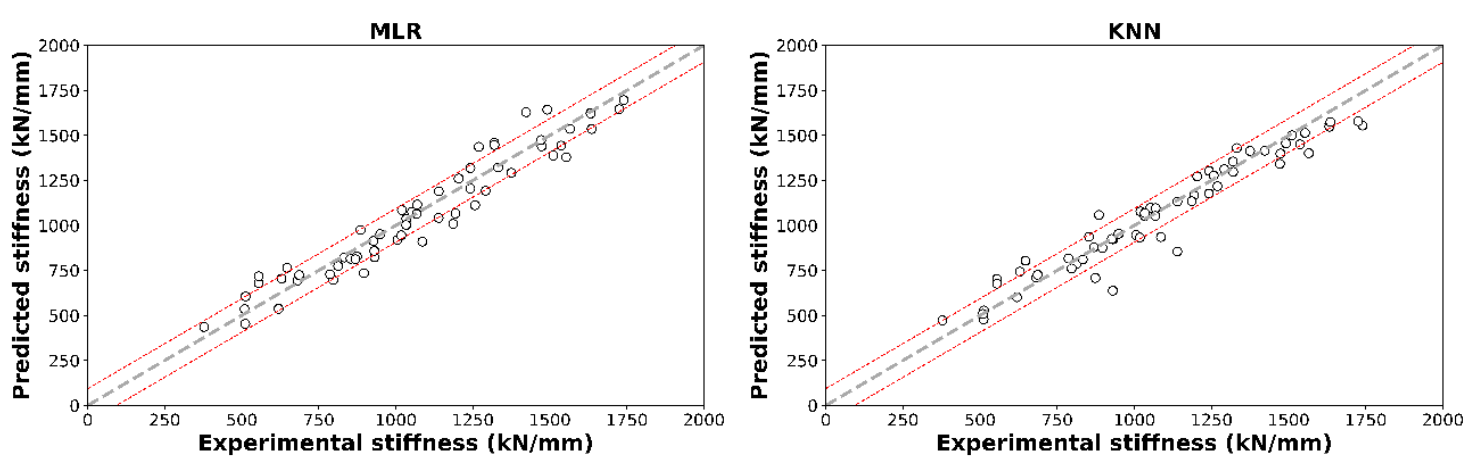

RT
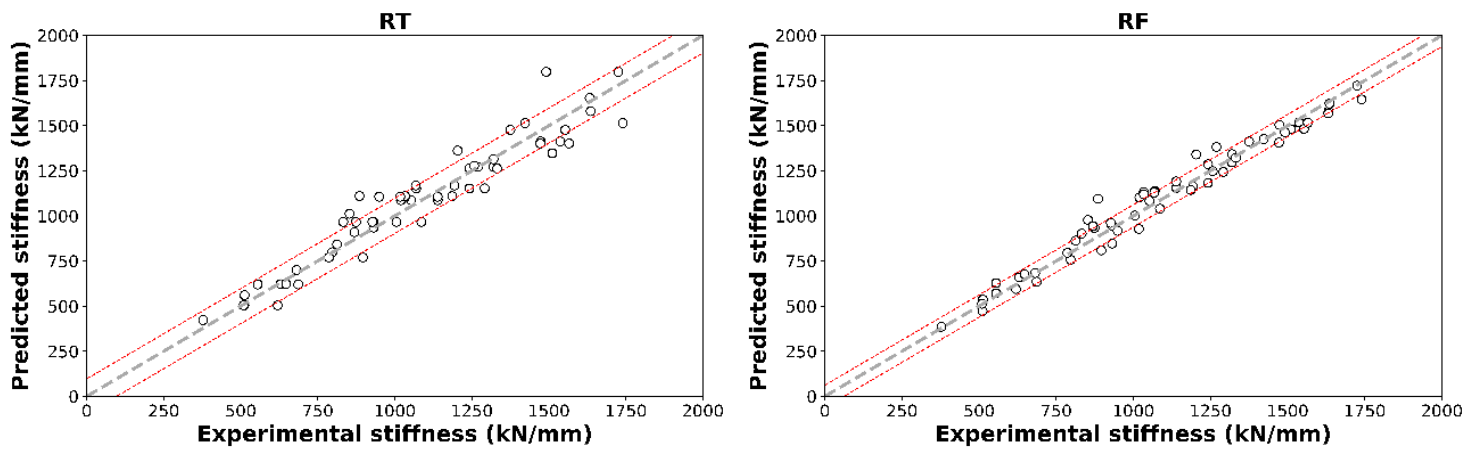

GB
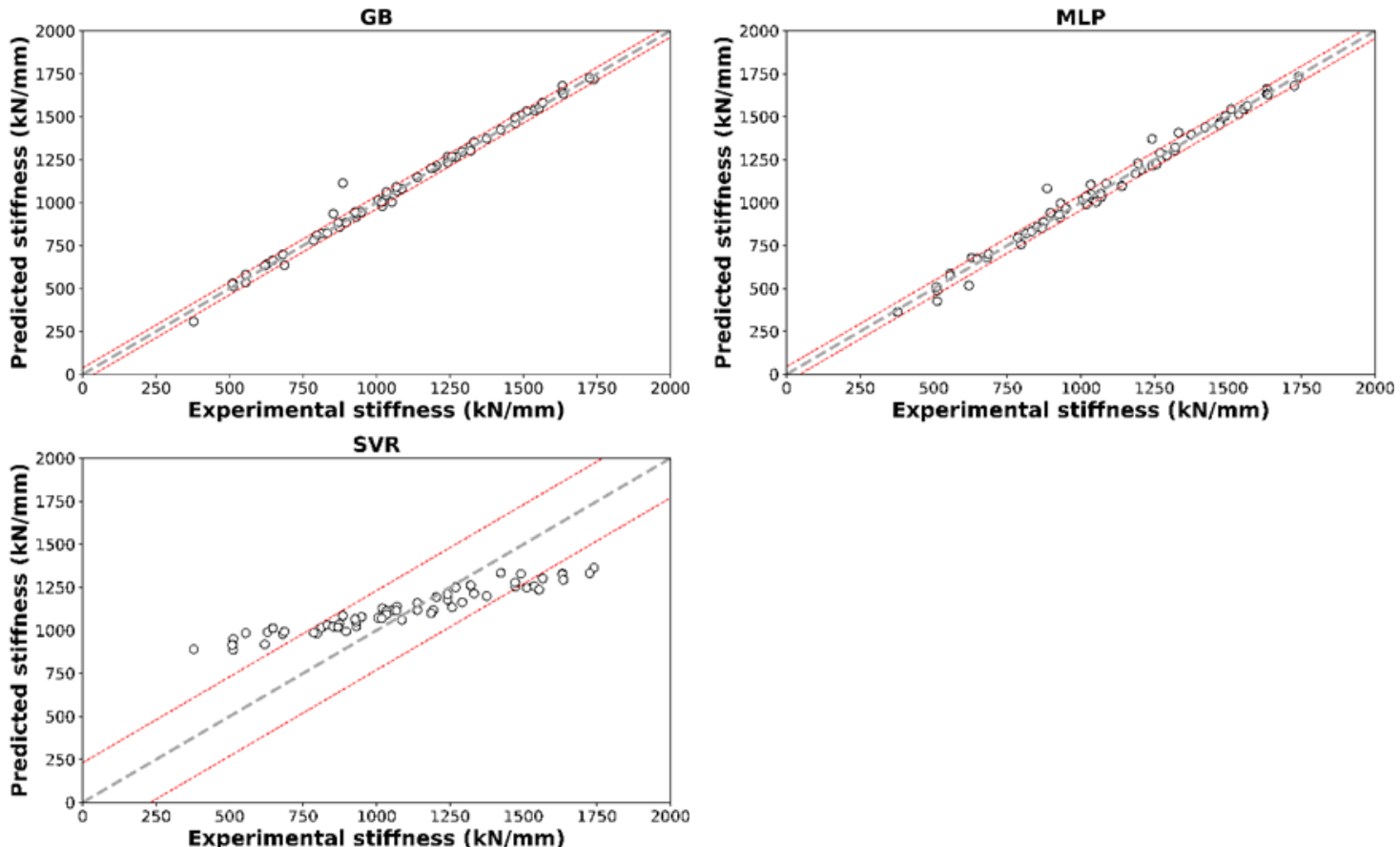

Figure 5.

\subsection{Partial Dependence Plots}

It is generally known how service variables (temperature, toe load, frequency and axle load) influence the dynamic stiffness of the rail pads. For higher axle loads, the stiffness is higher. This phenomenon is explained because, for high loads, the capacity of the rail pads to deform is lower and, therefore, the rail pads have a more rigid response. On the other hand, the higher the frequency, the greater the stiffness obtained. This is explained because, if the load frequency is high, the pads have less time to adapt to the movement and, therefore, less displacements are obtained. Regarding temperature, the higher it is, the greater is the capacity to deform and, 
therefore, the lower is the stiffness. Finally, in a similar way to what happens with the axle load, by increasing the toe load, the capacity of the pad to deform reduces and more rigid behaviour is observed.

As mentioned before, PDPs enable answers to be obtained about how and how much the influence of each of the variables is. Figure 6, Figure 7 and Figure 8 show the PDPs of the features for the three pads. In each of the graphs, a central trend thick line is represented, which is accompanied by a number of thin lines that provide a measure of the dispersion to the central line. The dark blue line is the average value of all generated cases, while the light blue ones are

From Figure 6 it is observed that the evolution of the EPDM pad stiffness with both amplitude and frequency are approximately proportional. In case of temperature, it can be seen that for very low temperatures there is a significant variation. Once $-20^{\circ} \mathrm{C}$ is exceeded, the effect of temperature is significantly reduced. Regarding the toe load effect, it is shown that there are two clearly differentiated phases. A first one up to approximately $12 \mathrm{kN}$ and another one from that value, where the influence of that toe load is accentuated.

301
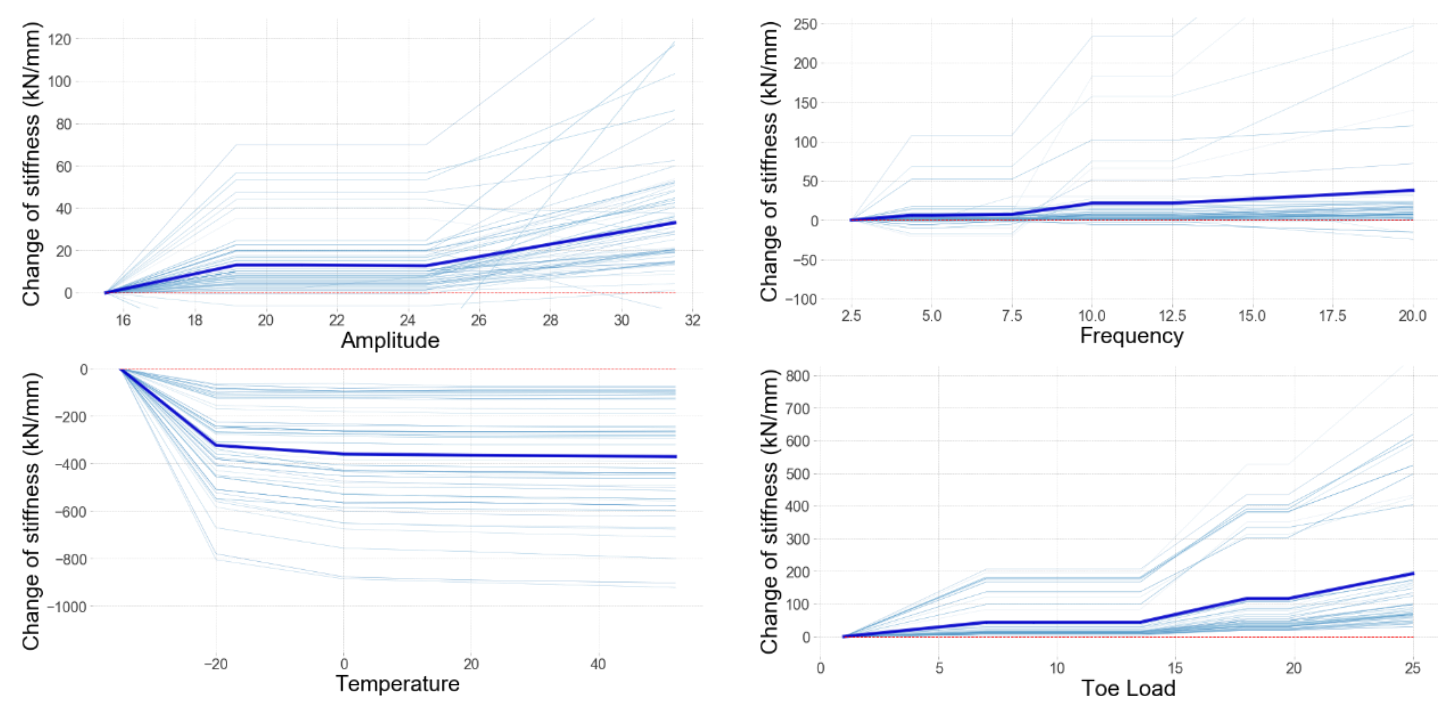
Figure 6. Partial dependence plots obtained on EPDM rail pads

303 Figure 7 shows that the effect on TPE pads of the increase in axle load, frequency and toe load is approximately proportional. In contrast, it can be seen that the influence of temperature is greater when the temperature is lower.
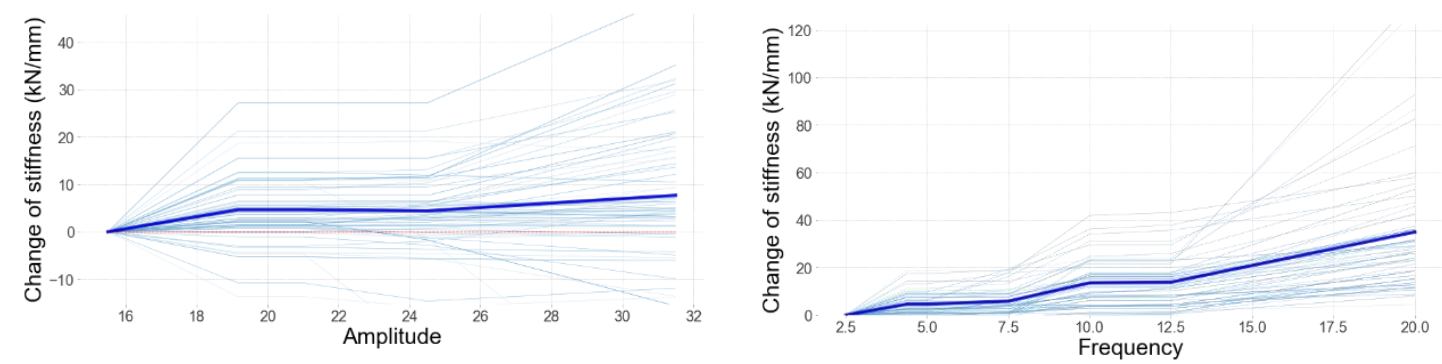

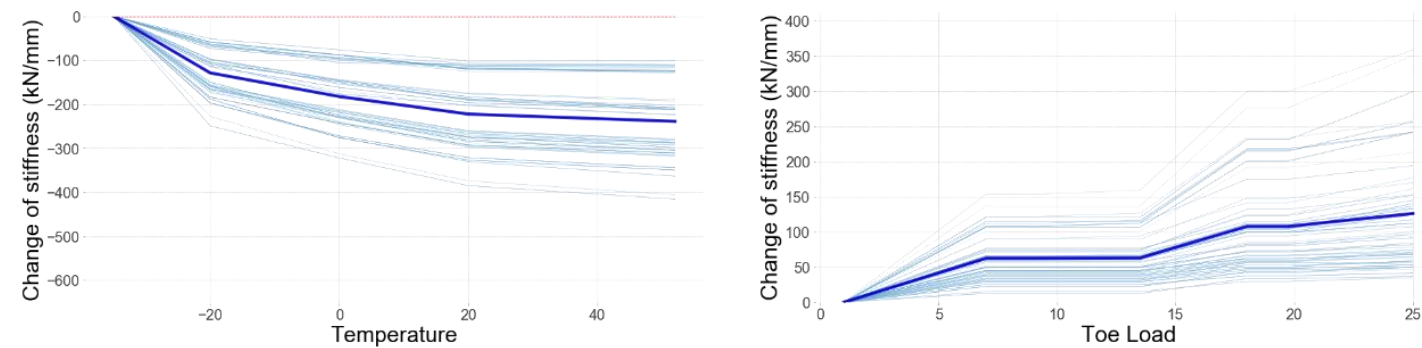

Figure 7. Partial dependence plots obtained on TPE rail pads

307

308

309

310

311

312

313

314

315

316

317

318

319

320

321

322

323

324

325

326

From Figure 8 it can be seen that the influence of amplitude, frequency and toe load on the EVA pad is approximately proportional within the range studied. It can be seen that the PDP corresponding to temperature does not seem to match with what could be expected, this is because the influence of temperature is so small that it is screened by the uncertainty of the measurement.
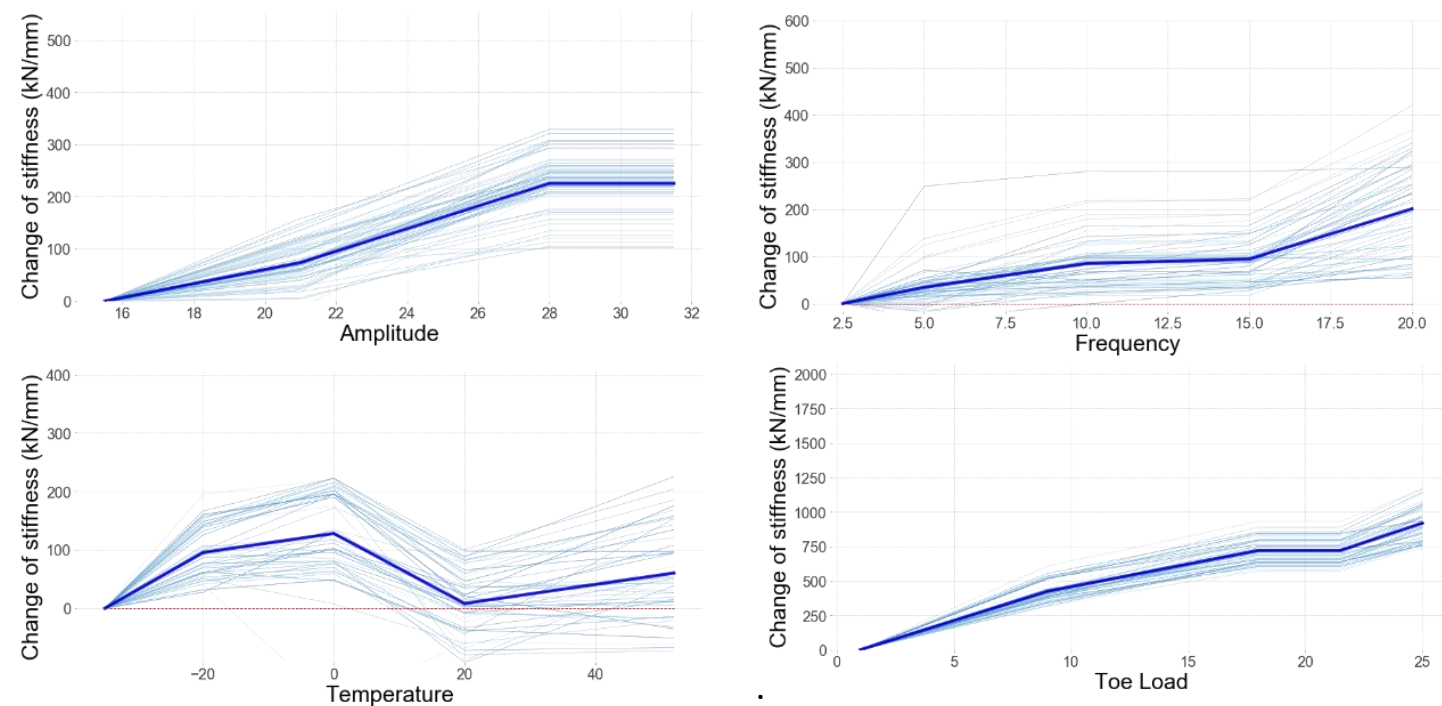

Figure 8.

Partial dependence plots obtained on EVA rail pads

\subsection{Feature and Permutation Importance}

The results obtained with $\mathrm{FI}$ and $\mathrm{PI}$ are summarized in Table 6 and represented as bar charts in Figure 9. Both algorithms display consistent results which is evidence of reliability, provided that the fundamentals on which $\mathrm{Fl}$ and $\mathrm{PI}$ are grounded are different and independent.

From the analysis of Figure 9, it is possible to determine which variables (temperature, toe load, frequency and axle load) have most influence on the pads' dynamic stiffness. For the EPDM pad, these parameters are temperature and toe load. Similarly, in the case of TPE, the parameters that have most influence on the stiffness are temperature and toe load. In the case of the EVA pad, the parameter with the greatest influence on the stiffness is temperature. It should be noted that the most influential parameters on pad behaviour are temperature and toe load and that the standard for characterizing these components only contemplates testing at different temperatures in extreme cases and does not propose any variation of the toe load.

Table 6. Quantitative relevance analysis parameter for each rail pad

\begin{tabular}{ccccccc}
\hline & \multicolumn{3}{c}{ Feature Importance } & \multicolumn{3}{c}{ Permutation Importance } \\
& EPDM & TPE & EVA & EPDM & TPE & EVA \\
\hline Temperature & $0.630 \pm 0.070$ & $0.690 \pm 0.040$ & $0.042 \pm 0.009$ & $1.430 \pm 0.370$ & $1.440 \pm 0.190$ & $0.088 \pm 0.009$
\end{tabular}



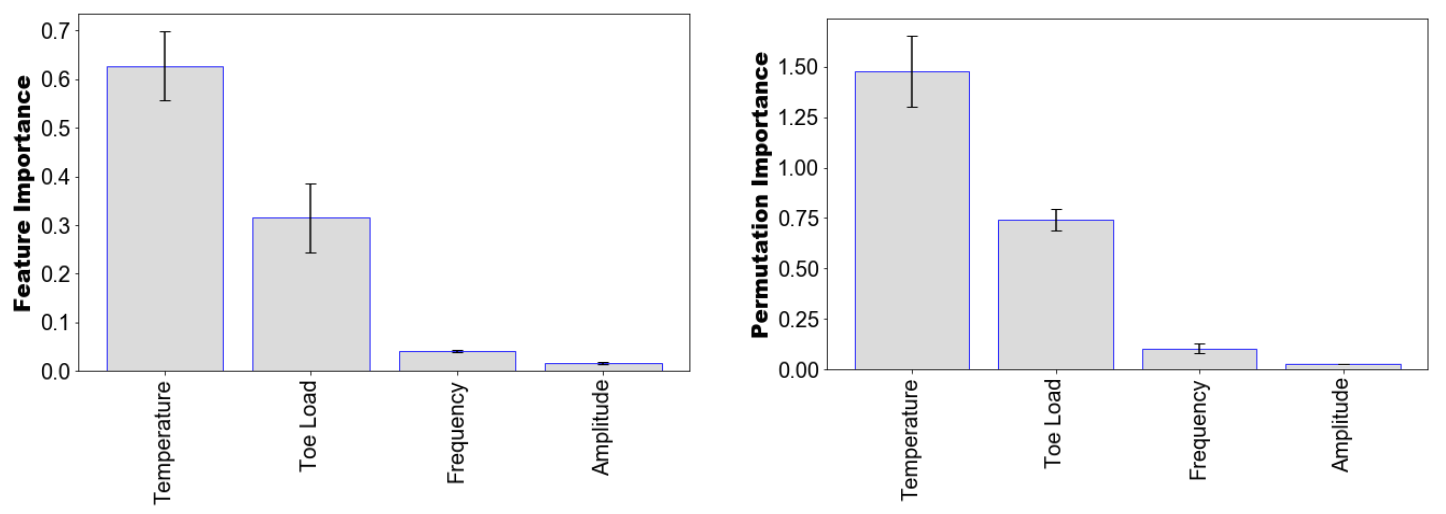

EPDM
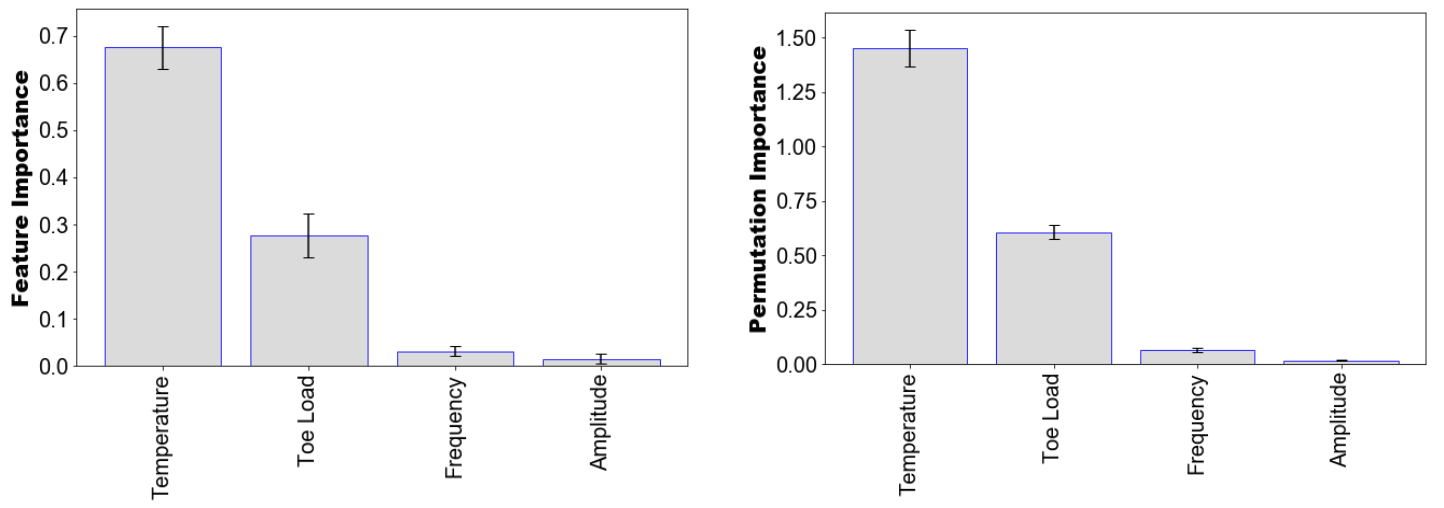

TPE
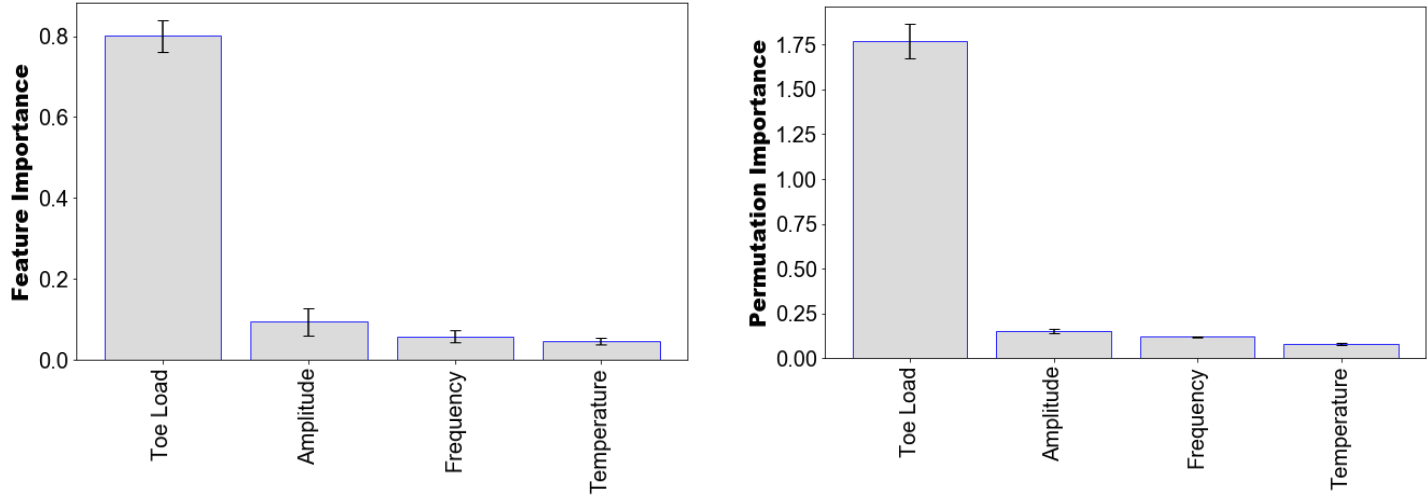

EVA

Figure 9. Quantitative relevance analysis parameter for each rail pad

4.4. Application for the implementation of the optimal ML algorithm

An application was created on the Microsoft .Net platform. The Visual Studio 2017 development environment was used with the $\mathrm{C \#}$ programming language. The user interface is based on Windows Forms. The operating requirements are a 64-bit computer with .Net Framework 4.7 or higher (for example, any 64-bit Windows 10 computer meets this requirement). Click Once technology was used to deploy and download this application via Web. This mechanism enables the app to be updated. The predictive machine learning model has been created using the ML.Net library. It is an open source multiplatform library [71]. The algorithm employed was an 

application can be downloaded from the following link: "http://www.cuartas.es/railpad/" in Microsoft Edge.

\section{5. Conclusions and Future Developments}

342 At present, there are no models, neither analytical nor numerical, that enable the dynamic stiffness of rail pads to be obtained depending on the assembly and operation conditions. However, this would be helpful because the polymeric materials used to manufacture these pads display a highly non-linear mechanical response and interactions among the service variables occur. Previous studies have experimentally obtained the influence of one or another of these variables. But this experimental information, scarce and dispersed, has not enabled reliable models to be produced.

The experimental scope of the present work includes a total of 720 dynamic tests carried out on three of the most common pad materials. The difficulties in modelling the response of these materials using conventional procedures justify the adoption of alternative data-driven methodologies. For this purpose, several machine learning algorithms are implemented and optimized. Gradient Boosting was found to be the optimal algorithm for the three materials. In general, Random Forest, which is also an ensemble method, and Multilayer Perceptron have provided very notable results. The ability of Gradient Boosting is highlighted by the coefficient of determination and mean absolute percentage error in the test dataset, which are $0.995 / 5.08 \%, 0.995 / 2.25 \%$ and $0.988 / 2.38 \%$, for EPDM, TPE and EVA, respectively.

Two alternative methodologies, feature importance and permutation importance, which enable the features to be sorted by order of importance, are also implemented. These procedures provide consistent results. Temperature and toe load are the variables that most influence the dynamic stiffness of EPDM and TPE, while toe load is the most relevant feature for EVA. Overall, toe load is the most influential parameter, regardless of the material tested. This result is particularly remarkable since the standards that define the experimental conditions for determination of the dynamic stiffness of rail pads do not take it into account. In the authors' opinion, the experimental and analytical evidence provided by this study is sufficient to motivate a possible update of the standards currently in force.

This information is useful for track design and for providing information about the maintenance procedures to the infrastructure managers. In addition, the algorithms developed here can be easily coupled with finite element models that include all the track components. This would enable the determination of the overall impact of the variables considered here, temperature, axle load, toe load and frequency on the infrastructure behaviour. These developments would support the optimization of the maintenance tasks and contribute to increasing the durability of tracks.

374 Considering the potential interest of the results of this research for the railway industry, an 375 application implementing the optimal Machine Learning algorithm was developed. This application enables the prediction of the dynamic stiffness of the rail pads covered in this study (manufactured either in EPDM, TPE or EVA) from the operating conditions. 


\section{Acknowledgements}

This work was supported by FCT, through IDMEC, under LAETA, project UIDB/50022/2020.

386

387

\section{Formatting of funding sources}

\footnotetext{
This research did not receive any specific grant from funding agencies in the public, commercial, or not-for-profit sectors.
}

\section{References}
[1] International Energy Agency. The Future of Rail. 2019.
[2] Iwnicki SD, Spiryagin M, Cole C, McSweeney T. Handbook of Railway Vehicle Dynamics. 2nd ed. CRC Press; 2019. https://doi.org/10.1201/9780429469398.

[3] Esveld C. Modern Railway Track. MRT Prod 2001.

[4] Yang J, Zhu S, Zhai W. A novel dynamics model for railway ballastless track with mediumthick slabs. Appl Math Model 2020;78:907-31. https://doi.org/10.1016/J.APM.2019.09.051.

[5] Sainz-Aja J, Pombo J, Tholken D, Carrascal I, Polanco J, Ferreño D, et al. Dynamic calibration of slab track models for railway applications using full-scale testing. Comput Struct 2020;228:106180. https://doi.org/10.1016/j.compstruc.2019.106180.

[6] Peltokangas O, Nurmikolu A. Evolution of Railway Track Settlement after Ballast Tamping. Int J Railw Technol 2015;4:1-18. https://doi.org/10.4203/ijrt.4.2.1.

[7] Bosso N, Gugliotta A, Zampieri N. A Comprehensive Strategy to Estimate Track Condition and its Evolution. Int J Railw Technol 2012;1:1-19. https://doi.org/10.4203/ijrt.1.2.1.

[8] Mezher SB, Connolly DP, Woodward PK, Laghrouche O, Pombo J, Costa PA. Railway critical velocity - Analytical prediction and analysis. Transp Geotech 2016;6:84-96. https://doi.org/10.1016/j.trgeo.2015.09.002.

[9] Indraratna B, Nimbalkar S, Rujikiatkamjorn C. Modernisation of Rail Tracks for Higher Speeds and Greater Freight. Int J Railw Technol 2013;2:1-20. https://doi.org/10.4203/ijrt.2.3.1.

[10] Fortunato E, Paixão A, Calçada R. Railway Track Transition Zones: Design, Construction, Monitoring and Numerical Modelling. Int J Railw Technol 2013;2:33-58. https://doi.org/10.4203/ijrt.2.4.3.

[11] Antunes P, Magalhães $\mathrm{H}$, Ambrósio J, Pombo J, Costa J. A co-simulation approach to the wheel-rail contact with flexible railway track. Multibody Syst Dyn 2019;45. https://doi.org/10.1007/s11044-018-09646-0.

[12] Muñoz S, Aceituno JF, Urda P, Escalona JL. Multibody model of railway vehicles with weakly coupled vertical and lateral dynamics. Mech Syst Signal Process 2019;115:57092. https://doi.org/10.1016/j.ymssp.2018.06.019.

[13] Pombo J, Ambrósio J, Silva M. A new wheel-rail contact model for railway dynamics. Veh Syst Dyn 2007;45:165-89. https://doi.org/10.1080/00423110600996017. 
[14] Alonso A, Guiral A, Gimenez JG. Wheel Rail Contact: Theoretical and Experimental Analysis. Int J Railw Technol 2013;2:15-32. https://doi.org/10.4203/ijrt.2.4.2.

[15] Pombo J, Ambrósio J. Application of a wheel-rail contact model to railway dynamics in small radius curved tracks. Multibody Syst Dyn 2008;19:91-114. https://doi.org/10.1007/s11044-007-9094-y.

[16] Marques F, Magalhães H, Pombo J, Ambrósio J, Flores P. A three-dimensional approach for contact detection between realistic wheel and rail surfaces for improved railway dynamic analysis. Mech Mach Theory 2020;149:103825. https://doi.org/10.1016/j.mechmachtheory.2020.103825.

[17] Sichani MS, Enblom R, Berg M. Non-Elliptic Wheel-Rail Contact Modelling in Vehicle Dynamics Simulation. Int J Railw Technol 2014;3:77-96. https://doi.org/10.4203/ijrt.3.3.5.

[18] Magalhães H, Marques F, Liu B, Antunes P, Pombo J, Flores P, et al. Implementation of a non-Hertzian contact model for railway dynamic application. Multibody Syst Dyn 2019. https://doi.org/10.1007/s11044-019-09688-y.

[19] Marques F, Magalhães H, Liu B, Pombo J, Flores P, Ambrósio J, et al. On the generation of enhanced lookup tables for wheel-rail contact models. Wear 2019;434-435:202993. https://doi.org/10.1016/j.wear.2019.202993.

[20] Bowe CJ, Mullarkey TP. Wheel-rail contact elements incorporating irregularities. Adv Eng Softw 2005;36:827-37. https://doi.org/10.1016/j.advengsoft.2005.03.026.

[21] Arslan MA, Kayabaşi O. 3-D Rail-Wheel contact analysis using FEA. Adv Eng Softw 2012;45:325-31. https://doi.org/10.1016/j.advengsoft.2011.10.009.

[22] Vollebregt EAH, Steenbergen MJMM. A Methodology for Assessing Track Irregularities with respect to Rail Damage. Int J Railw Technol 2015;4:85-105. https://doi.org/10.4203/ijrt.4.4.5.

[23] Pombo J, Ambrósio J. An alternative method to include track irregularities in railway vehicle dynamic analyses. Nonlinear Dyn 2012;68:161-76. https://doi.org/10.1007/s11071-011-0212-2.

[24] Hsu SS, Fagan N. Improving Switches and Crossings Performance and Reliability. Int J Railw Technol 2016;5:79-93. https://doi.org/10.4203/ijrt.5.3.4.

[25] Coleman I, Kassa E, Smith R. Wheel-Rail Contact Modelling within Switches and Crossings. Int J Railw Technol 2012;1:45-66. https://doi.org/10.4203/ijrt.1.2.3.

[26] Hölscher P. The Dynamics of Foundations for High Speed Lines on Soft Soils. Int J Railw Technol 2012;1:147-66. https://doi.org/10.4203/ijrt.1.1.7.

[27] Sañudo R, Markine V, Pombo J. Study on Different Solutions to Reduce the Dynamic Impacts in Transition Zones for High-Speed Rail. J Theor Appl Vib Acoust 2017;3:199-222. https://doi.org/10.22064/tava.2018.80091.1095.

[28] Iwnicki SD, Bevan AJ. Damage to Railway Wheels and Rails: A Review of the Causes, Prediction Methods, Reduction and Allocation of Costs. Int J Railw Technol 2012;1:12146. https://doi.org/10.4203/ijrt.1.1.6.

[29] Pombo J. Application of a Computational Tool to Study the Influence of Worn Wheels on Railway Vehicle Dynamics. J Softw Eng Appl 2012;05:51-61. https://doi.org/10.4236/jsea.2012.52009. 
[30] Stichel S, Jönsson P-A, Casanueva C, Hossein Nia S. Modelling and Simulation of Freight Wagon with Special attention to the Prediction of Track Damage. Int J Railw Technol 2014;3:1-36. https://doi.org/10.4203/ijrt.3.1.1.

[31] Kuka N, Verardi R, Ariaudo C, Pombo J. Impact of maintenance conditions of vehicle components on the vehicle-track interaction loads. Proc Inst Mech Eng Part C J Mech Eng Sci 2018;232:2626-41. https://doi.org/10.1177/0954406217722803.

[32] Pita AL. Infraestructuras ferroviarias. EDICIONES UPC; 2006.

[33] Dahlberg TLE. On the use of under-sleeper pads in tracks with varying track stiffness. Proc. - 9th Int. Heavy Haul Conf. "Heavy Haul Innov. Dev., 2009.

[34] Pita AL, Teixeira PF, Robuste F. High speed and track deterioration: The role of vertical stiffness of the track. Proc Inst Mech Eng Part F J Rail Rapid Transit 2004;218:31-40. https://doi.org/10.1243/095440904322804411.

[35] Sol-Sánchez M, Moreno-Navarro F, Rubio-Gámez MC. The use of elastic elements in railway tracks: A state of the art review. Constr Build Mater 2015;75:293-305. https://doi.org/10.1016/j.conbuildmat.2014.11.027.

[36] Ferreño D, Casado J, Carrascal IA, Diego S, Ruiz E, Saiz M, et al. Experimental and finite element fatigue assessment of the spring clip of the SKL-1 railway fastening system. Eng Struct 2019;188:553-63. https://doi.org/10.1016/j.engstruct.2019.03.053.

[37] Pombo J, Almeida T, Magalhães $H$, Antunes P, Ambrósio J. Finite Element Methodology for Flexible Track Models in Railway Dynamics Applications. Int J Veh Struct Syst 2013;5. https://doi.org/10.4273/ijvss.5.2.01.

[38] Varandas JN, Paixão A, Fortunato E, Hölscher P, Calçada R. Numerical Modelling of Railway Bridge Approaches: Influence of Soil Non-Linearity. Int J Railw Technol 2014;3:73-95. https://doi.org/10.4203/ijrt.3.4.4.

[39] Sainz-Aja J, Carrascal I, Polanco JA, Thomas C, Sosa I, Casado J, et al. Self-compacting recycled aggregate concrete using out-of-service railway superstructure wastes. J Clean Prod 2019;230. https://doi.org/10.1016/j.jclepro.2019.04.386.

[40] Sainz-Aja J, Carrascal I, Polanco JA, Thomas C. Fatigue failure micromechanisms in ed aggregate mortar by $\mu C T$ analysis. J Build Eng 2019:101027. https://doi.org/10.1016/J.JOBE.2019.101027.

[41] Zhu S, Cai C, Luo Z, Liao Z. A frequency and amplitude dependent model of rail pads for the dynamic analysis of train-track interaction. Sci China Technol Sci 2015;58:191-201. https://doi.org/10.1007/s11431-014-5686-y.

[42] Fenander Å. Frequency dependent stiffness and damping of railpads. Proc Inst Mech Eng Part F J Rail Rapid Transit 1997;211:51-62. https://doi.org/10.1243/0954409971530897.

[43] Wei K, Wang F, Wang P, Liu ZX, Zhang P. Effect of temperature- and frequency-dependent dynamic properties of rail pads on high-speed vehicle-track coupled vibrations. Veh Syst Dyn 2017;55:351-70. https://doi.org/10.1080/00423114.2016.1267371.

[44] Wei K, Zhang P, Wang P, Xiao J, Luo Z. The Influence of Amplitude- and FrequencyDependent Stiffness of Rail Pads on the Random Vibration of a Vehicle-Track Coupled System. Shock Vib 2016;2016:1-10. https://doi.org/10.1155/2016/7674124.

[45] Wei K, Zhao Z, Ren J, Ou L, Wang P. High-speed vehicle-slab track coupled vibration analysis of the viscoelastic-plastic dynamic properties of rail pads under different 
preloads and temperatures. Veh

Syst

Dyn

$2019 ; 3114$.

https://doi.org/10.1080/00423114.2019.1673444.

509

510

511

512

513

514

515

516

517

518

519

520

521

522

523

524

525

526

527

528

529

530

531

532

533

534

535

536

537

538

539

540

541

542

543

544

545

546

[46] Cukrowicz KC, Jahn DR, Graham RD, Poindexter EK, Williams RB. Suicide risk in older adults: Evaluating models of risk and predicting excess zeros in a primary care sample. J Abnorm Psychol 2013;122:1021-30. https://doi.org/10.1037/a0034953.

[47] Kaewunruen S, Remennikov AM. Response and Prediction of Dynamic Characteristics of Worn Rail Pads Under Static Preloads. Univ Wollongong 2007:9-12.

[48] Kaewunruen S, Remennikov AM. Laboratory Measurements of Dynamic Properties of Rail Pads under Incremental Preload. 19th Australas. Conf. Mech. Struct. Mater., Christchurch, New Zealand: Taylor \& Francis; 2007, p. 319-24.

[49] Kiani J, Camp C, Pezeshk S. On the application of machine learning techniques to derive seismic fragility curves. Comput Struct 2019;218:108-22. https://doi.org/10.1016/j.compstruc.2019.03.004.

[50] Kawamura K, Miyamoto A. Condition state evaluation of existing reinforced concrete bridges using neuro-fuzzy hybrid system. Comput Struct 2003;81:1931-40. https://doi.org/10.1016/S0045-7949(03)00213-X.

[51] Gou J, Fan Z-W, Wang C, Guo W-P, Lai X-M, Chen M-Z. A minimum-of-maximum relative error support vector machine for simultaneous reverse prediction of concrete components. Comput Struct 2016;172:59-70. https://doi.org/10.1016/J.COMPSTRUC.2016.05.003.

[52] Basudhar A, Missoum S. Adaptive explicit decision functions for probabilistic design and optimization using support vector machines. Comput Struct 2008;86:1904-17. https://doi.org/10.1016/J.COMPSTRUC.2008.02.008.

[53] Ekevid T, Wiberg NE. Wave propagation related to high-speed train a scaled boundary FE-approach for unbounded domains. Comput Methods Appl Mech Eng 2002;191:394764. https://doi.org/10.1016/S0045-7825(02)00341-9.

[54] EN 13481-2:2012+A1. Railway Applications. Track. Performance Requirements for Fastening Systems. Fastening Systems for Concrete Sleepers 2017.

[55] EN 13146-9:2011+A1. Railway Applications. Track. Test Methods for Fastening Systems. Part 9: Determination of the Stiffness 2012.

[56] Sainz-Aja JA, Carrascal IA, Ferreño D, Pombo J, Casado JA, Diego S. Influence of the operational conditions on static and dynamic stiffness of rail pads. Mech Mater 2020;148.

[57] Welcome to Python.org n.d.

[58] NumPy - NumPy n.d.

[59] pandas - Python Data Analysis Library n.d.

[60] scikit-learn: machine learning in Python - scikit-learn 0.22.2 documentation n.d.

[61] Matplotlib: Python plotting - Matplotlib 3.2.0 documentation n.d.

[62] seaborn: statistical data visualization - seaborn 0.10 .0 documentation n.d.

[63] Asaithambi S. Why, How and When to Scale your Features - GreyAtom - Medium. Medium 2017. 
[64] Vapnik V, Chervonenkis A. A note on one class of perceptrons. Autom Remote Control 1964;25.

[65] Feng Y, Gao W, Wu D, Tin-Loi F. Machine learning aided stochastic elastoplastic analysis. Comput Methods Appl Mech Eng 2019;357:1-31. https://doi.org/10.1016/j.cma.2019.112576.

[66] Mohamed AE. Comparative Study of Supervised Machine Learning Techniques for Intrusion Detection 2007;14:5-10. https://doi.org/10.15546/aeei-2014-0021.

[67] Boser BE, Guyon IM, Vapnik VN. A training algorithm for optimal margin classifiers. Proc Fifth Annu Work Comput Learn Theory - COLT '92 1992:144-52. https://doi.org/10.1145/130385.130401.

[68] Zhang $Y$, Wen Z, Pei H, Wang J, Li Z, Yue Z. Equivalent method of evaluating mechanical properties of perforated Ni-based single crystal plates using artificial neural networks. Comput Methods Appl Mech Eng 2020. https://doi.org/10.1016/j.cma.2019.112725.

[69] Guido S, Müller A. Introduction to Machine Learning with Python. A Guide for Data Scientists. O’Reilly Media; 2016.

[70] Mierzwa-Sulima O. "Please, explain." Interpretability of black-box machine learning models. Appsilon DATA Sci 2019.

[71]. NET machine learning \& artificial intelligence. Microsoft n.d.

[72] FastTreeRegressionTrainer Class (Microsoft.ML.Trainers.FastTree) | Microsoft Docs. Microsoft n.d.

[73] Rashmi K V., Gilad-Bachrach R. DART: Dropouts meet Multiple Additive Regression Trees. ArXivOrg 2015. 